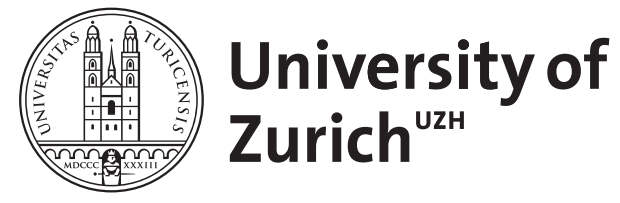

\title{
Klima auf Gegenseitigkeit
}

Leist, Anton

DOI: https://doi.org/10.1515/jfwe.2012.215

Posted at the Zurich Open Repository and Archive, University of Zurich

ZORA URL: https://doi.org/10.5167/uzh-50605

Book Section

Published Version

Originally published at:

Leist, Anton (2011). Klima auf Gegenseitigkeit. In: Birnbacher, Dieter; Honnefelder, Ludger; Sturma, Dieter. Klimawandel und intergenerationelle Gerechtigkeit. Berlin: Walter de Gruyter, 159-178.

DOI: https://doi.org/10.1515/jfwe.2012.215 


\title{
Klimaschutz im Spannungsfeld von intergenerationeller Gerechtigkeit und demokratischer Legitimität
}

\author{
von Peter Rinderle
}

\begin{abstract}
Der anthropogene Klimawandel stellt die heutigen Demokratien vor eine neue Bewährungsprobe. Angesichts der weithin unbezweifelten Tatsache, dass der Klimawandel zukünftigen Generationen großen Schaden zufügen wird, stellt sich die Frage, ob die Legitimität demokratischer Institutionen nicht in einem Widerstreit zu den Forderungen der intergenerationellen Gerechtigkeit steht: Wäre der intergenerationellen Gerechtigkeit durch die politische Herrschaft von wissenschaftlichen Experten nicht viel eher gedient? Oder sollten wir umgekehrt von einem Primat der Demokratie gegenüber Erwägungen der intergenerationellen Nachhaltigkeit ausgehen? Gibt es die Möglichkeit, das Mehrheitsprinzip mit der intergenerationellen Gerechtigkeit zu versöhnen? Ist vielleicht eine defizitäre politische Verwirklichung der normativen Idee der Demokratie für Verletzungen der intergenerationellen Gerechtigkeit verantwortlich? Darf man eventuell sogar von einer Kompatibilität oder gar einer Konvergenz der intergenerationellen Gerechtigkeit und der Realisierung einer echten Gestalt von Demokratie ausgehen? Diese Fragen möchte ich in diesem Überblicksaufsatz klären und einer ersten Antwort zuführen.
\end{abstract}

Keywords: Klimawandel, intergenerationelle Gerechtigkeit, Demokratie, Tragik der Allmende.

\section{Einleitung}

Der anthropogene Klimawandel kann als eine Form der intergenerationellen Ungerechtigkeit verstanden werden: Wir - und in erster Linie sind damit die Menschen in den westlichen Industrieländern gemeint - verletzen durch unsere Emission von Treibhausgasen die Rechte zukünftiger Personen auf eine Sicherung ihrer elementaren Lebensgrundlagen. Können wir uns gegen diese moralische Kritik mit dem Hinweis zur Wehr setzen, dass in Demokratien schließlich einer Mehrheit der Bürger das Recht auf politische Herrschaft zukomme und die Klimapolitik der westlichen Industrieländer daher demokratisch legitimiert sei? Oder könnten die Mitglieder zukünftiger Generationen erwidern, die Forderungen der intergenerationellen Gerechtigkeit (kurz: IG) seien von größerer Bedeutung als die Idee der demokratischen Legitimität (kurz: DL)? Könnten sie gar mit guten Gründen die Einsetzung einer Öko-Diktatur verlangen? Stellen die mit den Folgen des Klimawandels verbundenen Verletzungen der IG also die Idee einer DL in Frage? Oder ist vielleicht 
eher eine defizitäre Gestalt der Demokratie für die Verletzung der Standards der IG verantwortlich? Und darf man dann nicht eher von einer Kompatibilität oder gar einer Konvergenz der IG und der Realisierung einer echten Gestalt der Demokratie ausgehen? Diese Fragen möchte ich in diesem Überblicksaufsatz klären und - so weit wie möglich - einer ersten Antwort zuführen. Ich beginne mit einer kritischen Präsentation der Inkompatibilitätsthese und lote dann nach und nach verschiedene Möglichkeiten einer Kompatibilität bzw. auch Konvergenz von IG und DL aus.

\section{Ist die Demokratie mit der Klimaverantwortung überfordert?}

Bereits in den 70er Jahren des letzten Jahrhunderts waren verschiedene Autoren der Auffassung, die demokratische Form politischer Herrschaft sei mit der Lösung des Problems des kollektiven Handelns nicht zu vereinbaren. ${ }^{1}$ Als Einstieg in unsere speziellere Thematik bietet sich das jüngste Buch The Climate Change Challenge and the Failure of Democracy von David Shearman und Joseph Smith an. Ihre zentrale These lautet, dass die Demokratie keine überzeugende Antwort auf den Klimawandel finden und deshalb unserer Verantwortung gegenüber zukünftigen Generationen nicht gerecht werden könne.

Sehen wir uns ihre Thesen und Argumente im Einzelnen an. Eine erste These der Autoren lautet, die Demokratie sei in ökologischer Hinsicht problematisch, weil sie selbst eine Ursache des Problems sei. Die liberale Demokratie, so Shearman und Smith, sei „ecologically flawed as a social system because it leads to the tragedy of the commons".2 Die Mehrheit der Mitglieder einer Gemeinschaft schiele notwendig immer auf den eigenen Vorteil, die ,Tragik der Allmende“ sei daher schon im Herzen der Demokratie angelegt. ${ }^{3}$ Aus der Sicht einer einzelnen Person sei unkooperatives Verhalten demnach zwar immer rational, nur habe die individuelle Rationalität eine besondere Form der kollektiven Irrationalität zur Folge, die früher (etwa beim Gefangenendilemma) oder später (beim Klimawandel) auch Nachteile für alle beteiligten Personen haben werde. Shearman und Smith stützen diese These mit dem folgenden Argument: Die Demokratie biete alle Freiheiten der Welt, ermögliche es jedem Individuum, unbegrenzten Reichtum zu akkumulieren und sei aufs Engste

\footnotetext{
HARDIN 1968; HeIlBRONER 1974; OpHuls 1977.

SHEARMAN, SMITH 2007, 11; meine Hervorh.

HARDIN 1968. Siehe auch JONAS 1979, 55; BIRNBACHER 1988, 258-268 sowie THOMPSON 2005, 246: „Democracies are systematically biased in favor of the present.“ LEGGEWIE und WELZER meinen ähnlich, „,der kurze Zeittakt demokratischer Politik [läuft] einer langfristigen und nachhaltigen Problemlösung chronisch zuwider“ (2010, 157); Demokratien „,vernachlässigen [...] systematisch Zukunft“ (ibid., 159).
} 
mit dem Kapitalismus verbunden: „Liberal capitalism and democracy have fused together." "Im Rahmen der kapitalistischen Logik sei die Tragik der Allmende nicht nur unausweichlich; die Demokratie trage vielmehr selbst zur Zerstörung der Umwelt bei.

Mit einer zweiten These spielen Shearman und Smith auf eine Begleiterscheinung der Demokratie an, die mit einer in ihren Augen höchst fragwürdigen Vorstellung des guten Lebens zu tun hat: Die Wertschätzung der individuellen Freiheit in zeitgenössischen Demokratien sei eng mit einer hedonistisch orientierten, auf Konsum, ja Gier ausgerichteten Massenkultur verbunden. ${ }^{5}$ Damit gehen Shearman und Smith natürlich über eine bloße Beschreibung bestimmter politischer Organisationsformen hinaus und stellen die Demokratie in einen unmittelbaren Zusammenhang mit der freiheitlich-individualistischen Lebensweise des modernen Menschen. Bei ihrem Plädoyer für eine Ablösung der Demokratie durch eine autoritäre Form der politischen Herrschaft stellen sie ausdrücklich die besondere Wertschätzung der Freiheit in Frage und behaupten, ohne die Integrität ökologischer Systeme ,values such as freedom and autonomy make no sense at all“. ${ }^{6} \mathrm{Da}$ es mir allein auf die mögliche Vereinbarkeit eines politischen Entscheidungsmechanismus mit den Forderungen der IG ankommen soll, kann hier die Frage außer Betracht bleiben, ob die Demokratie mit anderen Kulturphänomenen Hand in Hand geht, eventuell sogar auf einem fragwürdigen Anthropozentrismus beruht und durch eine alternative, ökozentrische Vision ersetzt werden sollte. ${ }^{7}$

Eine dritte und wesentlich moderatere These der Autoren lautet, die Demokratie könne einfach nur die ökologische Krise nicht bewältigen. Sie sei „unable to defend the commons" 8 und daher auch nicht dazu imstande, einen effektiven und intergenerationell gerechten Klimaschutz zu realisieren. Demokratische Institutionen, so Shearman und Smith, ,are not suited to deal with crises care situations“. ${ }^{9}$ Diese dritte These sollte man nun sorgfältig von der ersten These unterscheiden: Die Demokratie könnte nämlich tatsächlich eine Ursache für die ökologische Krise sein, gleichzeitig aber über das Potenzial verfügen, einen Ausweg aus ihr zu finden; umgekehrt könnte die Demokratie einfach ,nur' unfähig sein, einen Ausweg aus einer Krise zu finden, die nicht von ihr verursacht wurde. Und obwohl die erste These zunächst radikaler erscheinen mag, enthält nur die dritte These eine echte Inkompatibilitätsannahme: Die Demokratie mag den Klimawandel (mit-)verursacht haben (These 1), doch bedeutet das nicht, dass sie nicht zu einem effektiven Schutz des Klimas beitragen könnte. Denn nur wenn die Demokratie keinen Ausweg aus der ökologi-

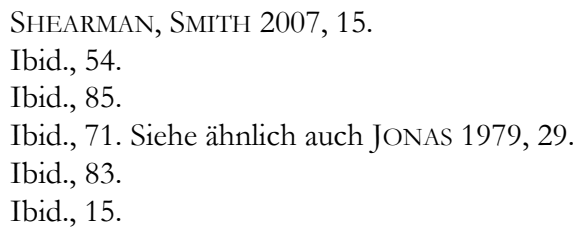


schen Krise aufzeigen kann (These 3), scheint es notwendig, über eine effektivere Alternative nachzudenken.

\section{Wäre ein autoritäres Regime besser geeignet?}

Angesichts der möglicherweise katastrophalen Folgen des Klimawandels und angesichts der allgemein zu beobachtenden Unfähigkeit der gegenwärtigen Politik stehen wir vor der Frage, ob demokratische Institutionen überhaupt in der Lage sind, dieser Herausforderung auf angemessene Weise zu begegnen. Shearman und Smith kommen zu einem negativen Ergebnis und schlagen eine „Platonic form of authoritarianism based upon the rule of scientific experts " als Alternative vor. ${ }^{10}$ Sollten wir demokratische Verfahren also durch autoritäre Institutionen ersetzen, die uns (intergenerationell) gerechte Entscheidungen garantieren können? Gegen diese Idee kann man zunächst den allgemeinen Einwand geltend machen, dass es in einer pluralistischen Gesellschaft nicht nur einen vernünftigen Dissens über rivalisierende Konzeptionen des guten Lebens gibt, die Geister sich vielmehr auch an den Inhalten der Gerechtigkeit scheiden. Diese Meinungsverschiedenheiten lassen sich nicht immer auf kognitive Defizite, auf Vorurteile oder Tendenzen zur Parteilichkeit zurückführen. ${ }^{11}$ Gleichermaßen kompetente und ehrliche Personen können also durchaus zu unterschiedlichen und dennoch vernünftigen Interpretationen hinsichtlich der Forderungen der Gerechtigkeit kommen.

Das gilt natürlich auch für die möglichen Inhalte einer Konzeption der IG bzw. der Klimagerechtigkeit. Zahlreiche Fragen bilden heute den Gegenstand einer heftigen Kontroverse: ${ }^{12}$ Welche Folgen wird der Klimawandel tatsächlich haben? Wie sind diese Folgen in wirtschaftlicher und moralischer Hinsicht genau zu bewerten? Und welche politischen Maßnahmen sind zur Stabilisierung des Klimas wirklich geeignet? Es kann dabei nicht wundernehmen, dass man auch zu unterschiedlichen Auffassungen hinsichtlich der Forderungen des Standpunkts der Unparteilichkeit kommt. Sicher: Diese Auffassungen können ihren Ursprung jeweils in Vorurteilen oder im jeweiligen Selbstinteresse haben. Doch eine Person, die für sich eine besondere moralische Expertise in Anspruch nimmt, wird man heute eher mit Misstrauen begegnen. Da die Gerechtigkeitsvorstellungen einer Person zum Teil immer auch von deren eigenen Interessen beeinflusst sein werden und diese reflektieren ${ }^{13}$, kann

10 Ibid., 2. Zu den Grenzen der demokratischen Mehrheitsherrschaft und den Vorteilen einer Tyrannis, ,die in unserem Zusammenhang eben eine wohlwollende, wohlinformierte und von der richtigen Einsicht beseelte Tyrannis sein muß“, vgl. weiterhin JONAS 1979, 262 f.

11 Dazu: Rawls 2003, 128-131.

12 Vgl. Nordhaus 2008; STERN 2009.

13 Christiano 2008, 59. 
niemand ein Monopol auf die richtige Interpretation der Unparteilichkeit für sich in Anspruch nehmen.

Welche Auswirkungen hat dieser Konflikt, und wie lässt er sich schlichten? Zunächst kann man folgende Schlussfolgerung ziehen: Da die Forderungen der Gerechtigkeit umstritten sind, eignet sich eine bestimmte Konzeption der Gerechtigkeit nur begrenzt für die Begründung der Legitimität politischer Institutionen. ${ }^{14}$ Die Forderungen der Gerechtigkeit können von verschiedenen Personen unterschiedlich ausgelegt werden; der Staat aber beansprucht ein Monopol für die Festlegung und Durchsetzung verbindlicher Regeln des Zusammenlebens und duldet auf diesem Feld keinen Rivalen neben sich. ${ }^{15}$ Für die Schlichtung dieses Streits gibt es nun verschiedene Möglichkeiten. Hobbes hat etwa die Errichtung eines Leviathans vorgeschlagen, der alle Macht in einem Gemeinwesen in seinen Händen bündeln und dann auch eine autoritative Interpretation der Forderungen der Gerechtigkeit vornehmen kann. Mit dem demokratischen Mehrheitsprinzip steht uns heute eine weitere Möglichkeit zur Schlichtung dieses Streits zur Verfügung. Da das Mehrheitsprinzip nur die Legitimität politischer Herrschaft begründen soll, muss es den Dissens über Gerechtigkeitsfragen nicht antasten. Über die Gerechtigkeit mögen die Geister weiterhin auseinander gehen; politisch legitim ist, was die Mehrheit entscheidet. Das Majoritätsprinzip weist den großen Vorteil auf, mit dem Faktum des modernen Pluralismus vereinbar zu sein. ${ }^{16}$

Auf unser spezielles Problem übertragen bedeutet das: Auch die Forderungen der IG sind umstritten. ${ }^{17}$ Es wäre daher unzulässig, das Recht auf politische Herrschaft - etwa eines elitären Kreises von Experten - auf eine besondere moralische Einsicht zu gründen. Nachdem es vernünftige Meinungsverschiedenheiten über die Inhalte der IG gibt, haben wir keinen guten Grund, demokratische Verfahren durch ein autoritäres Regime zu ersetzen. Damit will ich nicht sagen, dass die Inhalte der IG auf demokratischem Wege ermittelt werden könnten; denn ich meine, es gibt durchaus verfahrensunabhängige Standards zur Bewertung der Ergebnisse eines demokratischen Verfahrens. ${ }^{18}$ Das bedeutet auch, dass diese Ergebnisse unter Umständen ungerecht sein können und sich selbst eine Mehrheit aller betroffenen Personen in Fragen der Gerechtigkeit täuschen kann. Dennoch verfügen wir mit dem Mehrheitsverfahren über eine Möglichkeit, dem Konflikt verschiedener Gerechtigkeitstheorien aus dem Weg zu gehen und gleichzeitig einige minimale und weithin unumstrittene Forderungen der Gerechtigkeit in die politische Realität umzusetzen.

Immerhin kann das demokratische Verfahren für sich in Anspruch nehmen, die Freiheit und Gleichheit der Bürger eines Gemeinwesens zum Ausdruck zu brin-

14 Dazu allgemein: RiNDERLE 2005, 213-283.

Vgl. Christiano 2008, 78.

16 Dazu: Waldron 1999, 99; Rinderle 2011.

17 Zur Debatte: Birnbacher 1988; Tremmel 2006; Gosseries, Meyer 2009.

18 Dazu: Goodin 2004; Gutman, Thompson 2004, 23-26; Christiano 2008, 97. 
gen. ${ }^{19}$ Insofern kommen demokratische Institutionen - auch wenn sie ungerechte Entscheidungen treffen - zumindest einer Elementarforderung der Gerechtigkeit nach. Und auf dem uns interessierenden Terrain des Klimawandels verhält es sich nicht viel anders: Nachdem es gerade auch hier viele Kontroversen über die Gerechtigkeit gibt, wäre die Ablösung demokratischer Verfahren durch die autoritäre Herrschaft besonderer moralischer oder wissenschaftlicher Experten nur eine Scheinlösung. Der Konflikt zwischen IG und DL kann in anderer Form an einer anderen Stelle - etwa zwischen den Forderungen der Gerechtigkeit und der politischen Legitimität wissenschaftlicher Experten - wieder auftauchen.

Sicher gibt es einen wichtigen Unterschied: Ein demokratisches Verfahren drückt - und auch das nur im Idealfall - vielleicht die Freiheit und Gleichheit der Mitglieder eines Gemeinwesens aus; die Mitglieder zukünftiger Generationen haben jedoch in politischen Verfahren der Gegenwart kein Partizipationsrecht. Wir stehen also vor einer grundlegenden Asymmetrie: Demokratische Verfahren mögen eine Lösung für unterschiedliche Gerechtigkeitsauffassungen von Zeitgenossen anzubieten haben. Doch so lange die Mitglieder zukünftiger Generationen kein Stimmrecht bei Entscheidungen haben, die sie betreffen, kann man auch nicht von der angemessenen Berücksichtigung der Freiheit und Gleichheit aller gegenwärtigen und auch zukünftigen Menschen sprechen.

Da ich auf dieses Problem der demokratischen Inklusion hier allerdings nicht näher eingehen kann, ${ }^{20}$ begnüge ich mich mit dem Hinweis, dass wir mit einem demokratischen Verfahren zumindest die Möglichkeit haben, die unterschiedlichen Vorstellungen zur IG der gegenwärtig lebenden Bürger auf eine faire Weise zu berücksichtigen. An dieser Stelle bricht die Symmetrie zum allgemeinen Verhältnis von Gerechtigkeit und Demokratie also noch nicht auf. Und was dann die mögliche Asymmetrie zu den Auffassungen der Mitglieder zukünftiger Generationen angeht, die in den gegenwärtigen politischen Verfahren keine Stimme haben, können wir uns im Augenblick auf die Hoffnung stützen, dass ihre Auffassungen im breiten Spektrum der gegenwärtigen Kontroversen bereits abgedeckt sind. Ob ihnen auch ein Recht zur politischen Partizipation bei gegenwärtigen Entscheidungen zukommt, ja ob die Ausübung eines solchen Rechts überhaupt vorstellbar ist und die Rede von einer ,intergenerationellen Demokratie، sinnvoll ist - das sind schwierige Fragen, denen ich hier nicht nachgehen kann. ${ }^{21}$

19 Dazu: RiNDERLE 2011.

20 Dazu: JOHNSON 2007; BECKMAN 2009, 23-61.

21 Dazu: Holden 2002, 93-99; Johnson 2007; BeCKMAN 2009, 167-187. 


\section{Empirische Belege?}

Der Umstand, dass es weitreichende und dabei durchaus vernünftige Meinungsverschiedenheiten über die genaueren Inhalte unserer Verantwortung gegenüber zukünftigen Generationen geben kann, lässt erhebliche Zweifel darüber aufkommen, ob ein autoritärer Diktator, mit dem Anspruch, sich auf eine besondere moralische oder wissenschaftliche Expertise zu stützen, tatsächlich für die effektive Durchsetzung eines gerechten Klimaschutzes geeignet ist. Abgesehen davon, dass die Selektion eines weisen und zugleich moralisch integren Diktators mit erheblichen Schwierigkeiten verbunden ist, kann man die intellektuellen bzw. sittlichen Kompetenzen einer Person ohnehin nicht als Grundlage für einen Anspruch auf die Ausübung politischer Herrschaft gelten lassen. ${ }^{22}$ Darüber hinaus ist zu beachten: Die wissenschaftliche Expertise tritt heute selbst im Plural auf. So gibt es nicht nur mehrere Autoren, die für eine Demokratisierung der wissenschaftlichen Expertise plädieren $^{23}$; die Vertreter einer epistemischen Theorie der Demokratie vertreten zudem die Auffassung, demokratischen Verfahren wohne selbst eine Tendenz inne, zu richtigen Ergebnissen zu führen: ${ }^{24}$ Die Betroffenen könnten die Folgen politischer Entscheidungen selbst am besten einschätzen; selbst einem wohlwollenden Diktator würde also ein großer Teil des für die Qualität politischer Entscheidungen notwendigen Wissens gar nicht zur Verfügung stehen. Deshalb gibt es auch aus der Perspektive einer expertokratischen Sichtweise gute Gründe dafür, der Mehrheit der Bürger das letzte Wort zu geben.

Gehen wir jetzt einen Schritt weiter: Selbst wenn wir unsere Meinungsverschiedenheiten in Bezug auf eine gerechte Klimapolitik beiseite legen und uns auf bestimmte Inhalte der IG einigen könnten, wäre damit immer noch kein hinreichender Grund gegeben, eine grundsätzliche Unvereinbarkeit der Forderungen der IG und den Grundlagen der DL zu postulieren. Nehmen wir einmal an, wir hätten eine klare und unumstrittene Vorstellung der Forderungen der IG im Allgemeinen und der Klimagerechtigkeit im Besonderen. Können wir daraus tatsächlich ein Legitimationsdefizit demokratischer Regime ableiten?

Bevor wir eine Antwort auf diese Frage geben können, sollten wir zwei Dinge unterscheiden. Man könnte die These vertreten, die heute existierenden Demokratien kämen ihrer Zukunftsverantwortung nur in einem unbefriedigenden Maße nach. Ein Demokrat könnte dann konzedieren, dass die sich ,demokratisch“ nennenden Regime dieser Verantwortung nicht gerecht werden; er könnte aber gleichzeitig behaupten, dieser Umstand spreche zunächst nur gegen den möglicherweise undemokratischen Charakter dieser Regime, nicht gegen die normative Idee der Demokratie

${ }^{22}$ Zur Kritik des expertokratischen Fehlschlusses vgl. HOLDEN 2002, 30-57; ESTLUND 2008, 3-5.

23 Vgl. z.B. NOWOTNY 2003.

24 ESTLUND 2008, 8. 
als solche. Vielleicht bleiben die real existierenden Demokratien weit hinter dem Ideal einer wirklichen Demokratie zurück. Würde man die politische Wirklichkeit nur im Sinne des Ideals verändern und eine ecbte Demokratie etablieren, ließe sich auch der Konflikt mit den Forderungen der Gerechtigkeit beseitigen. Auch für die klimapolitischen Unzulänglichkeiten der aktuellen Politik könne daher nicht die Idee der Demokratie selbst verantwortlich gemacht werden. Eine echte Demokratisierung der nationalstaatlichen Politik sowie eine Anwendung der demokratischen Idee in transnationalen, globalen oder intergenerationellen Kontexten böten vielmehr große Chancen, einen effektiven und gerechten Klimaschutz zu gewährleisten. Eine Untersuchung der Vereinbarkeit von IG und DL muss sich also mit zwei ganz verschiedenen Fragen auseinandersetzen: Gibt es, erstens, eine ideale und dennoch politisch realisierbare Gestalt der Demokratie, die den Forderungen der IG nachkommen könnte? Und sind, zweitens, die realen Demokratien in der Lage, für einen effektiven und gerechten Klimaschutz zu sorgen?

Was zunächst die erste Frage angeht, so hängt die Möglichkeit einer Vereinbarkeit der idealen Demokratie mit den Forderungen der Gerechtigkeit in erster Linie von den genauen Inhalten dieses Ideals ab. Dazu gibt es heute eine unübersichtliche Anzahl verschiedener Vorschläge, die von einer Vertiefung der demokratischen Ideale - etwa durch umfangreichere und inklusivere Partizipations- und Deliberationsmöglichkeiten ${ }^{25}$, durch ,,innovative Formen direkter Beteiligung“26 - über verschiedene Strategien der Selbst- und der Fremdbindung ${ }^{27}$, der Beschränkung von Mehrheitsentscheidung und der Implementierung von indirekten, repräsentativen Formen der Demokratie ${ }^{28}$ bis hin zur Erweiterung demokratischer Verfahren in neuen Kontexten - etwa auf einer transnationalen, globalen ${ }^{29}$ oder gar intergenerationellen Ebene $^{30}$ - reichen. Und selbst wenn man die Frage nach den Möglichkeiten der Umsetzung dieser Ideale in die politische Realität vorläufig beiseitelässt, bleibt bei diesen Vorschlägen immer noch die Frage offen, ob sie ihren Anspruch, etwa einen gerechteren Klimaschutz bewerkstelligen zu können, einlösen können.

Ohne hier eine skeptische Position vertreten zu wollen, möchte ich in diesem Zusammenhang nur darauf hinweisen, dass man diesen Anspruch nicht auf dem Wege der begrifflichen Analyse des betreffenden Ideals, sondern nur durch empirische Belege begründen wird können. Und es liegt in der Natur der Sache, dass es schwierig sein dürfte, für diese Zwecke aussagekräftige empirische Daten zu gewinnen. Die These, eine Vertiefung und/oder Erweiterung des demokratischen Ideals in den gegenwärtigen politischen Realitäten könne den Widerstreit zwischen den Forderungen der IG und der Idee der DL ausräumen, muss man daher zur Zeit

${ }^{25}$ Vgl. ECKERSLEY 2004; JOHNSTON 2007.

${ }^{26}$ LEgGewie, Welzer 2010, 149.

27 BIRNBACHER 1988, 261; BIRNBACHER 2009, 292-297.

28 BiRnBACHER 1988, 263f.; THOMPSON 2005.

29 TÄNNSJÖ 2008; Bummel 2010.

${ }^{30}$ HoLdEN 2002, 58-111. 
noch als ungesichert ansehen. Aber das gilt auch für die gegenteilige Auffassung: Auch der Skeptiker kann sich nicht einfach mit dem Hinweis auf die klimapolitischen Defizite der existierenden Demokratien begnügen.

\section{Methodische Grenzen von Systemvergleichen}

Wir wissen also nicht, ob eine bessere Inkarnation der Idee der Demokratie mehr für eine Stabilisierung des Klimas leisten könnte, als es die gegenwärtig existierenden Demokratien tun. Schon die Untersuchung dieser Frage ist mit nicht unerheblichen Schwierigkeiten verbunden, da wir mit sozialen Institutionen nicht wie mit chemischen Substanzen experimentieren können. Würde die Einführung umfangreicher Partizipationsmöglichkeiten der Bürger tatsächlich zu einer besseren Berücksichtigung der Rechte und Interessen zukünftiger Generationen führen? ${ }^{31}$ Würde ein demokratisch organisierter Weltstaat die gegenwärtigen Sorgen der Menschheit tatsächlich hintanstellen, wenn es um die Verwirklichung einer anspruchsvollen Konzeption der IG geht? Im vorliegenden Kontext bedürfte vor allem das folgende Problem einer Lösung: Ist allein durch eine globale Erweiterung demokratischer Verfahren die Gegenwartsfixierung von Mehrheitsentscheidungen bzw. der kurze Zeittakt demokratischer Politik in den Griff zu bekommen? Die Frage, warum ausgerechnet eine globale Form der Demokratie eine höhere Zukunftsfähigkeit für sich in Anspruch nehmen kann als traditionelle, nationalstaatliche Formen der Demokratie, wird oft gar nicht gestellt - geschweige denn beantwortet.

Vielleicht ist es angesichts dieser Schwierigkeiten einfacher, eine klimapolitische Bilanz für die real existierenden Institutionen aufzustellen: Wir müssten zu diesem Zweck ja nur, so die Vorstellung, die Klimapolitik von demokratischen und autoritären Regimen nebeneinander stellen und dann miteinander vergleichen. Das Ergebnis dieses Vergleichs werde uns über die jeweiligen Vor- und Nachteile der betreffenden Legitimationsformen in Bezug auf die Forderungen einer Konzeption der IG belehren. (Der Einfachheit halber vernachlässige ich hier den Umstand, dass es unterschiedliche Formen sowohl demokratischer als auch autoritärer Formen der Legitimität gibt.) Nur gibt es in der gegenwärtigen Debatte kein klares und eindeutiges Bild der Verhältnisse. Eine Mehrheit der Autoren scheint allerdings einen für eine demokratisch legitimierte Klimapolitik zuletzt recht positiven Schluss zu ziehen: Die umweltpolitische Bilanz von Demokratien könne sich trotz aller Probleme

31 Zu entsprechenden Zweifeln vgl. BEESON 2010, 281: „The reality is that the Philippines, the country with arguably the most vibrant civil society in Southeast Asia, also has one of the most appalling environmental records." 
sehen lassen, der Vergleich zwischen Demokratien und autoritären Regimen falle jedenfalls nicht eindeutig zum Nachteil der ersteren aus. ${ }^{32}$

Selbst wenn man aber den existierenden Demokratien eine hohe umweltpolitische Kompetenz zubilligen könnte, würde das nicht ausreichen, um die Forderung nach einer Vertiefung und Erweiterung realer Demokratien im Sinne einer idealen Gestalt der Demokratie zurückzuweisen. Eine positive umweltpolitische Bilanz allein würde daher nicht für die politische Legitimation der aktuellen Demokratien ausreichen. Eine zufriedenstellende Wirklichkeit ist schließlich kein hinreichendes Argument gegen das Vorliegen besserer Möglichkeiten, und sie ist deshalb auch niemals eine hinreichende Grundlage für die Zurückweisung der Auffassung, eine Reform der aktuellen Institutionen würde viele Verbesserungen erlauben und sei deshalb eine Bedingung für die Legitimität politischer Institutionen. Aber die Belege reichen doch aus, um zumindest die klimapolitischen Vorzüge einiger Demokratien gegenüber manchen autoritären Regimen aufzuzeigen.

Man sollte dieses Resultat sicher nicht unterschätzen; es ist zumindest ein erstes Indiz, das für eine Vereinbarkeit von IG und DL spricht. Aber man sollte dieses Resultat in seiner Bedeutung für die vorliegende Problematik auch nicht überschätzen. Wenn wir nämlich die klimapolitische Bilanz zweier unterschiedlicher Regime vergleichen, müssen wir immer eine Abstraktion vornehmen und alle denkbaren Unterschiede der jeweiligen Kontexte dieser Regime ausblenden. Für einen echten Vergleich der klimapolitischen Vor- und Nachteile eines bestimmten Typs der Herrschaftsorganisation sind diese empirischen Vergleiche zuletzt nur von begrenzter Relevanz. Die Sozialwissenschaft kann uns beispielsweise die Auskunft erteilen, dass der Regimetyp D (D für Demokratie) eines bestimmten Staats X eine bessere umweltpolitische Bilanz aufweise als der Regimetyp A (A für Autokratie) eines Staats Y. Diese Auskunft ist für sich genommen natürlich nicht ohne Interesse, sie ist aber nicht mit der These identisch, dass D in X notwendig eine bessere Bilanz aufzuweisen habe als $\mathrm{A}$ in X. Zur Begründung dieser These müsste man eine reale Gegebenheit (Regimetyp D in X) mit einer hypothetischen Gegebenheit (Regimetyp A in X) vergleichen. Nun möchte ich nicht behaupten, dass ein solcher Vergleich grundsätzlich unmöglich ist. Dennoch birgt ein solches Vorgehen ganz eigene Schwierigkeiten; und außerdem sind die gegenwärtigen Untersuchungen - soweit ich das jedenfalls überblicken kann - weit davon entfernt, diesen strengen methodischen Anforderungen zu genügen.

Um ein einfaches Beispiel zu nennen: Wir können nicht einfach die (teilweise desaströse) Umweltpolitik der USA mit der (teilweise erfolgreichen) Umweltpolitik Chinas vergleichen. Wir müssten uns vielmehr die Frage vorlegen, ob ein autoritäres Regime in den USA nicht möglicherweise eine bessere umweltpolitische Bilanz vorweisen könnte als die real existierenden Institutionen in den US A. ${ }^{33}$ Und wir müssten uns dann zusätzlich fragen, ob die Einführung echter demokratischer Verfahren in

32 Vgl. GidDENS 2009, 73-90; HÖFFE 2009, 309-312.

33 Vgl. Friedman 2010, 483-512. 
China die umweltpolitische Bilanz dieses Staates entscheidend verbessern könnte. ${ }^{34}$ Die entscheidenden Fragen lauten also: Wäre die USA mit einem weisen und wohlwollenden Diktator in klimapolitischer Hinsicht vielleicht nicht sehr viel besser gefahren? Und hätte China umgekehrt mit der Beachtung der Normen der politischen Gleichheit nicht vielleicht eine sehr viel klimafreundlichere Politik betrieben?

So optimistisch also manche empirischen Belege für eine mögliche Vereinbarkeit eines effektiven und gerechten Klimaschutzes mit unseren Vorstellungen von der Legitimität demokratischer Institutionen stimmen mögen, so können umgekehrt andere empirischen Daten sowie die Ergebnisse mancher Gedankenexperimente einem allzu ungezügelten Enthusiasmus einen Dämpfer versetzen. Denn auch wenn empirische Belege für die Vor- und Nachteile bestimmter Formen der politischen Herrschaftsorganisationen nicht unmöglich sein dürften, setzt ihre Überzeugungskraft doch die Beachtung relativ anspruchsvoller methodischer Anforderungen voraus. Und nachdem nur der geringste Anteil der heutigen Diskussionsbeiträge diesen Anforderungen auf angemessene Weise tatsächlich Beachtung schenkt, bleibt ihre Bedeutung für die vorliegende Problematik begrenzt.

\section{Der intrinsische Wert demokratischer Verfahren}

In den letzten drei Abschnitten habe ich mich mit der Frage beschäftigt, ob ideale oder real existierende demokratische Institutionen ein angemessenes Mittel zum Zweck der Verwirklichung der Forderungen der IG sind. In einem letzten Schritt möchte ich über diese instrumentalistische Betrachtungsweise hinausgehen und auf die Möglichkeit eines intrinsischen Werts demokratischer Verfahren aufmerksam machen. Man kann sich zum einen - wie wir das hier bisher getan haben - fragen, ob die Ergebnisse und die empirischen Folgen demokratischer Verfahren bestimmte Vorteile haben. Man kann sich zum anderen aber auch die Frage vorlegen, ob wir ein Interesse an der in demokratischen Verfahren zum Ausdruck kommenden politischen Freiheit und Gleichheit aller Bürger haben - und zwar unabhängig von der Qualität ihres, outputs'.35

34 Vgl. BEESON 2010, 289: „In China [...] an authoritarian regime has arguably done more to mitigate environmental problems than any other government on earth $[\ldots]$ “. Als Beispiel verweist er auf die erfolgreiche Geburtenkontrolle in China.

35 Im Anschluss an den Ökonomen Bruno S. Frey unterscheiden LEGGEWIE und WELZER $(2010,172)$ einen verfahrensorientierten Prozessnutzen von einem outputorientierten „Ergebnisnutzen“ der Demokratie. Nur scheint ihnen an dieser Stelle zu entgehen, dass der demokratische Prozess auch - und zwar durch den „kurzen Zeittakt“, von dem sie selbst sprechen - eine Gefahr für die Klimastabilisierung darstellen kann. Warum eine „erfolgreiche Klimapolitik“ „mehr Demokratie“ (ibid., 173) erfordern sollte, bleibt daher ungeklärt. Der Reform der Demokratie gilt im Kapitel V. Die Große Transformation ohne- 
Auch der intrinsische Wert demokratischer Verfahren kann in einem engen Verhältnis zu bestimmten Vorstellungen der Gerechtigkeit gesehen werden. Nur wird man dann nicht mehr nur von einem kausal-instrumentalistischen Verhältnis sprechen können. Mein Vorschlag lautet, den intrinsischen Wert demokratischer Institutionen in ein ,expressives ${ }^{6}$ Verhältnis zu den Forderungen der Gerechtigkeit zu rücken. ${ }^{36}$ Man kann das allgemeine Wahlrecht und darüber hinausgehende Beteiligungsrechte als einen ,Ausdruck' der Werte der politischen Gerechtigkeit verstehen: Die Einführung des Wahlrechts steht nicht nur in einem letztlich immer nur kontingenten, empirischen Verhältnis zur Gerechtigkeit, das Wahlrecht bildet vielmehr ein unverzichtbares Ingredienz dessen, was wir überhaupt unter Gerechtigkeit verstehen.

Sicher kann es zu Konflikten zwischen Mehrheitsentscheidungen und den Forderungen der materialen Gerechtigkeit - und insbesondere natürlich den Forderungen der IG - kommen. ${ }^{37}$ Der Umstand allerdings, dass ein allgemeines Recht zur politischen Beteiligung selbst als eine Forderung einer (nun eher prozedural verstandenen) Gerechtigkeit verstanden werden kann, wird diese Konflikte doch in einem anderen Licht erscheinen lassen. Vor allem gebührt dann auch einer materialen Gerechtigkeit nicht unbedingt ein Vorzug gegenüber einer Idee der Demokratie, die eine prozedurale Gerechtigkeit in politischen Institutionen manifest macht. Wenn nämlich die Demokratie selbst als ein Ausdruck der Werte Freiheit und Gleichheit verstanden werden kann - und diese Werte bilden ja doch das Herzstück einer modernen Auffassung von Gerechtigkeit -, so können wir auch im spezielleren Kontext unserer Fragestellung von einer neuen Möglichkeit der Kompatibilität von IG und DL sprechen.

Zwar sollten wir im Rahmen des hier zur Debatte stehenden Problems nicht einfach von der sicherlich beschränkten Annahme ausgehen, die (real existierenden oder idealerweise möglichen) gegenwärtigen Demokratien könnten eine Idee der IG vollständig zum Ausdruck bringen. Aber wir können nun doch die Auffassung vertreten, dass eine Konzeption der IG - vor allem dann, wenn man sie auf eine kontraktualistische Grundlage stellt ${ }^{38}$ - unter anderem eine Forderung der Achtung der politischen Autonomie aller Generationen enthalten würde. Die Mehrheit unter den Mitgliedern der gegenwärtigen Generation kann sich somit also auf ein gutes Recht zur Beibehaltung demokratischer Institutionen berufen, so lange sie jedenfalls nicht

hin nicht mehr ihr primäres Interesse; dort vertreten sie nämlich die radikale These: „Eine nachhaltige Lösung der Probleme, die die Welt, wie wir sie kennen, hervorgebracht hat, erfordert nicht weniger als eine kulturelle Revolution [...]“ (ibid., 174; meine Hervorh.). Siehe dazu sehr viel vorsichtiger FRIEDMAN 2010, 256-258.

36 Ähnlich: GUTMAN, THOMPSON 2004, $21 \mathrm{f}$.

37 Vgl. wieder BEESON 2010, 282: „There is no compelling evidence that democracy of any sort will necessarily promote good environmental outcomes."

38 Dazu: BECKMAN 2008; RiNDERLE 2010. 
das entsprechende Recht einer Mehrheit der Mitglieder zukünftiger Generationen verletzt. ${ }^{39}$

Aus diesem Blickwinkel nimmt unser bisheriger Gedankengang eine interessante Wendung: Wir fragen uns nun nämlich nicht mehr nur, ob gegenwärtige demokratische Institutionen bestimmte Vorstellungen einer material verstandenen Gerechtigkeit verletzen. Wir müssen vielmehr auch die prozedurale Dimension der (intergenerationellen) Gerechtigkeit berücksichtigen. Unabhängig von den empirischen Folgen bestimmter Mehrheitsentscheidungen in der Gegenwart wird man darüber hinaus die Eingriffe in die für ein bestimmtes Regime konstituierenden Bestandteile in den Blick nehmen müssen. Schränkt also eine gegenwärtige Mehrheit die Möglichkeit zur Ausübung politischer Autonomie der Mitglieder zukünftiger Generationen ein, so wird man eine solche Entscheidung als einen Verstoß gegen die Forderungen der IG verstehen müssen - und zwar selbst dann, wenn man der gegenwärtigen Mehrheit weder den notwendigen wissenschaftlichen Sachverstand noch ein echtes Wohlwollen für zukünftige Generationen streitig machen kann. Wir haben es dann nämlich mit dem Phänomen eines intergenerationellen Paternalismus (bzw. gar einer intergenerationellen Tyrannei) zu tun, das bestimmte Freiheitsrechte der Mitglieder zukünftiger Generationen in Frage stellt. ${ }^{40}$

39 Dazu: WeIZsÄCKER 2010.

$40 \mathrm{Zu}$ den Spannungen zwischen der Nachhaltigkeit und der Demokratie siehe v. a. WEIZSÄCKER 2010, 1: „Es widerspräche dem Gedanken der Volkssouveränität, angewendet auf künftige Generationen, wenn die gegenwärtige Generation ihren Nachfolgern vorschreiben würde, welche Klimapolitik sie zu betreiben haben. Dies wäre, wenn es denn überhaupt gelänge, eine Usurpation von Entscheidungsrechten der Nachfolger durch die heutigen Entscheidungsträger. Eine Politik der Nachhaltigkeit, die künftige Generationen bindet, widerspricht somit dem Demokratieprinzip. [...] Die künftigen Generationen sind frei, die heutige Klimapolitik zu beenden und durch eine ganz andere Klimapolitik oder auch durch ein klimapolitisches Laisser-faire zu ersetzen.“ Weizsäckers These lautet daher: „Es gibt einen Primat des demokratischen Prinzips gegenüber dem Dogma der Nachhaltigkeit.“ (Ibid., 3) (Vgl. ähnlich JonAs 1979, 214; Hervorh. i. O., der allerdings von der Erhaltung der Möglichkeit der Staatskunst im allgemeinen spricht und dabei nicht-demokratische Formen der Staatskunst zumindest nicht explizit ausschließt: „[...] eine Verantwortung der Staatskunst ist, darauf zu achten, daß künftige Staatskunst möglich bleibt.“) Gerade weil ich Weizsäckers Abhandlung als einen wichtigen Beitrag zur gegenwärtigen Debatte ansehe, möchte ich vier kritische Anmerkungen machen: 1) Die Idee der Nachhaltigkeit ist kein Dogma, sie wird von vielen Autoren in Frage gestellt, und sie wird von sehr vielen Autoren mit guten Gründen verteidigt. 2) Zukünftige Generationen, das ist richtig, sind frei und haben ein Interesse an der Erhaltung und Ausübung ihrer (politischen) Autonomie. Ein dringlicheres Interesse wird allerdings ihrem Überleben - als einer Voraussetzung für die Ausübung ihrer Autonomie - gelten. 3) Es ist nicht richtig, dass die Klimapolitik gegenwärtiger Generationen künftige Generationen binden, eine Politik der Nachhaltigkeit also notwendig den Handlungsspielraum künftiger Generationen einschränken muss. Die Festlegung quanti- 
Umgekehrt gilt aber auch: Tastet die gegenwärtige Mehrheit das Recht zur Ausübung politischer Autonomie der Mitglieder zukünftiger Generationen nicht an, so wird man diese Selbstbeschränkung selbst dann noch als (prozedural) gerecht verstehen können, wenn sie (inhaltlich gesehen) verschiedene Wünsche offen lässt. Man könnte dann von einem intergenerationellen Liberalismus bzw. Libertarismus sprechen, der sich in erster Linie eine Gleichverteilung v. a. der politischen Rechte aller Generationen auf die Fahnen schreibt. Eine solche intergenerationelle Erweiterung des politischen Liberalismus kann selbst dann als ein echter Ausdruck der IG verstanden werden, wenn er in umwelt- und vor allem klimapolitischer Hinsicht weiterhin Defizite aufweist.

Die Vereinbarkeit von DL und IG wird also nicht schon allein deshalb in Frage gestellt, weil der Klimaschutz von demokratischen Mehrheiten manchmal auf sträfliche Weise vernachlässigt wird. Defizite beim Klimaschutz allein geben keinen Anlass dazu, die Legitimität demokratischer Institutionen auf grundlegende Weise in Frage zu stellen. Die Forderungen der IG gehen sicherlich nicht im Klimaschutz auf; die Aufgabe besteht vielmehr darin, die verschiedenen Dimensionen und Aspekte einer solchen intergenerationell erweiterten Konzeption der Gerechtigkeit vernünftig auszutarieren. ${ }^{41}$ Dabei ist dieses Ergebnis nicht als Rechtfertigung einer mangelnden Rücksichtsnahme gegenüber den Interessen der Mitglieder zukünftiger Generationen zu verstehen. Die Realisierung bestimmter Werte kann eben ihren Preis haben: Man kann daher auch das Recht einer Mehrheit zur politischen Autonomie anerkennen und gleichzeitig bedauern, dass die Entscheidungen in mancher Hinsicht - und zwar auch in moralischer Hinsicht - fehlerhaft sind. Allein die Durchführung eines demokratischen Verfahrens garantiert schließlich keine gerechten Entscheidungen. Umgekehrt sollten wir nicht ausschließen, dass ein autoritäres Regime eventuell tatsächlich die Forderungen der IG besser verwirklichen könnte.

tativer Emissionsziele kann von künftigen Mehrheiten natürlich revidiert werden. 4) Weizsäcker übersieht die Verpflichtungen der näheren künftigen Generationen gegenüber entfernteren künftigen Generationen. Sicherlich ist eine (nähere) künftige Generation frei, eine bestimmte Klimapolitik zu beenden oder zu revidieren. Doch genauso wie die gegenwärtige Generation gegenüber (näheren und entfernteren) künftigen Generationen in der Pflicht steht, steht auch die nähere künftige Generation gegenüber allen entfernteren künftigen Generationen in der Pflicht. Oder - und das ist mir nicht klar geworden - würde Weizsäcker einfach die Möglichkeit einer Konzeption der IG ablehnen?

41 Dazu: RinderLe 2010, 45. 


\section{Fazit}

Ziehen wir ein erstes, vorläufiges Fazit: Das Ziel meiner Überlegungen war eine Analyse des Spannungsfeldes von IG und DL im Hinblick auf politische Bemühungen um einen effektiven Klimaschutz. Das Ergebnis dieser Analyse lautet: Es gibt keinen guten Grund, von einer radikalen Inkompatibilität zwischen der Idee der DL und den Forderungen der IG zu sprechen. Auch eine Demokratie kann Vorsorge gegenüber der Wohlfahrt zukünftiger Personen treffen und einen effektiven Klimaschutz gewährleisten. Es gibt aber auch keinen Grund, sich in der naiven und optimistischen Hoffnung zu wiegen, wonach wir die Idee der Demokratie nur vollständig in die politische Wirklichkeit umsetzen und ihren Anwendungsbereich nur vertiefen bzw. erweitern müssten, um auch allen Forderungen der IG nachzukommen. Eine vollkommene Konvergenz oder Kongruenz dieser Kriterien gibt es genauso wenig wie eine radikale Inkompatibilität. Konflikte zwischen den Kernwerten der liberalen Demokratie und den Forderungen der IG bleiben nicht nur denkbar; sie werden ein unvermeidbares Merkmal unserer politischen Realität - und zwar gerade auch bei allen Versuchen eines wirksamen Klimaschutzes - bleiben. In solchen Fällen gilt es dann abzuwägen: Manchmal wird man den Werten der Demokratie den Vorzug geben, manchmal wird man aber auch den Interessen zukünftiger Personen einen Vorrang einräumen und die politische Legitimität demokratischer Verfahren qualifizieren und begrenzen müssen.

\section{Literatur}

BECKMAN, L. (2008): Do Global Climate Change and the Interest of Future Generations have Implications for Democracy?, in: Environmental Politics 17 (4), 610-624.

- (2009): The Frontiers of Democracy. The Right to Vote and its Limits, Basingstoke.

BEESON, M. (2010): The Coming of Environmental Authoritarianism, in: Environmental Politics 19 (2), 276-294.

BIRNBACHER, D. (1988): Verantwortung für zukünftige Generationen, Stuttgart.

- (2009): What Motivates us to Care for the (Distant) Future?, in: GossERIES, A., MEYER, L. (eds.), Intergenerational Justice, Oxford, 273-300.

Bummel, A., IGLESIAS, F., KERR, D. (2010): Democratizing Global Climate Policy through a United Nations Parliamentary Assembly, http:/ /www.kdun.org/resources/2010canberra.pdf (Stand: Dezember 2010).

Christiano, T. (2008): The Constitution of Equality. Democratic Authority and its Limits, New York. 
ECKersLey, R. (2004): The Green State: Rethinking Democracy and Sovereignty, Cambridge, Mass.

EsTLund, D.M. (2008): Democratic Authority. A Philosophical Framework, Princeton.

Friedman, T.L. (2010): Was zu tun ist. Eine Agenda für das 21. Jabrhundert, Frankfurt a. M.

GidDens, A. (2009): The Politics of Climate Change, Cambridge.

GOODIN, R.E. (2004): Democracy, Justice and Impartiality, in: DOWDING, K, GOODIN, R.E., Pateman, C. (eds.), Justice and Democracy. Essays for Brian Barry, Cambridge, 97-111.

Gutman, A., Thompson, D.F. (2004): Why Deliberative Democracy?, Princeton.

HARDIN, G. (1968): Tragedy of the Commons, in: Science 162, 1243-1248.

Heilbroner, R.L. (1974): An Enquiry into the Human Prospect, New York.

HÖFFE, O. (2009): Ist die Demokratie qukunftsfähig? Über moderne Politik, München.

Holden, B. (2002): Democracy and Global Warming, London.

JOHnson, G.F. (2007): Discursive Democracy in the Transgenerational Context and a Precautionary Turn in Public Reasoning, in: Contemporary Political Theory 6 (1), 67-85.

JONAS, H. (1979): Das Prinzip Verantwortung. Versuch einer Ethike für die technologische Zivilisation, Frankfurt a. M.

LEgGeWIE, C., WelZER, H. (2010): Das Ende der Welt, wie wir sie kannten. Klima, Zukunft und die Chancen der Demokratie, Bonn.

NordHAus, W.D. (2008): A Question of Balance, New Haven.

Nowotny, H. (2003): Democratising Expertise and Socially Robust Knowledge, in: Science and Public Policy 30 (3), 151-156.

OpHuLS, W. (1977): Ecology and the Politics of Scarcity, San Francisco.

RAWLS, J. (1975): Eine Theorie der Gerechtigkeit, Frankfurt a.M.

- (2003): Politischer Liberalismus, Frankfurt a.M.

RINDERLE, P. (2005): Der Zweifel des Anarchisten. Für eine neue Theorie von politischer Verpflichtung und staatlicher Legitimität, Frankfurt a.M.

- (2010): Climate Justice. A Contractualist Perspective, in: Analyse \& Kritik 32 (1), 39-61.

- (2011): Werte und Mechanismen der Demokratie, in: Zeitschrift für philosophische Forschung 65 (1), 70-91.

SheArman, D., Smith, J.W. (2007): The Climate Change Challenge and the Failure of Democracy, Westport.

STERN, N. (2009): Der Global Deal, München. 
TÄnnsJÖ, T. (2008): Global Democracy. The Case for a World Government, Edinburgh.

Thompson, D.F. (2005): Democracy in Time: Popular Sovereignty and Temporal Representation, in: Constellations 12 (2), 245-261.

TREMMEL, J. (ed.) (2006): Handbook of Intergenerational Justice, Cheltenham.

VAnderheiden, S. (2008): Atmospheric Justice. A Political Theory of Climate Change, Oxford.

WALDRON, J. (1999): Law and Disagreement, Oxford.

WEIZSÄCKER, C.C. VON (2010): Wie vertragen sich Nachbaltigkeit und Demokratie?, NZZ (20. Januar), http://www.nzz.ch/nachrichten/Hintergrund/dossiers/klimawandel/internationale_aspekte/wie_vertragen_sich_nachhaltigkeit_und_demokratie_1.4550282.html (Stand: Dezember 2010). 
Bereitgestellt von | UZH Hauptbibliothek / Zentralbibliothek Zürich Angemeldet Heruntergeladen am | 09.11.17 15:13 


\section{Dokumentation}


Bereitgestellt von | UZH Hauptbibliothek / Zentralbibliothek Zürich Angemeldet Heruntergeladen am | 09.11.17 15:13 